\title{
Assessing the influence of true hemolysis occurring in patient samples on emergency clinical biochemistry tests results using the VITROS ${ }^{\circledR} 5600$ Integrated system
}

\author{
SHUANGQING LIU ${ }^{1 *}$, JUAN LI $^{2 *}$, LI NING $^{1}$, DAWEI WU $^{1}$ and DIANJUN WEI ${ }^{3}$ \\ ${ }^{1}$ Department of Clinical Laboratory, The Second Hospital of Tianjin Medical University, Tianjin 300211; \\ ${ }^{2}$ Department of Rheumatology, Featured Medical Center of Chinese People's Armed Police Force, Tianjin 300072; \\ ${ }^{3}$ Department of Clinical Laboratory, Hebei Yanda Hospital, Langfang, Hebei 065201, P.R. China
}

Received June 11, 2021; Accepted August 24, 2021

DOI: $10.3892 /$ br.2021.1467

\begin{abstract}
Hemolysis is one of the most frequent causes of pre-analytical errors in the emergency department (ED), and it can lead to inaccurate blood results and often requires repeat testing. The purpose of the present study was to evaluate the effects of true hemolysis occurring in ED blood samples on routine clinical biochemistry tests using the VITROS ${ }^{\circledR} 5600$ Integrated system. A total of 92 pairs of blood samples were collected from 92 ED patients. Each pair of samples included one hemolyzed sample and one successful (non-hemolyzed) redraw from the same patient. A total of 21 common laboratory analytes and the hemolytic index (HI) were examined. The degree of hemolysis (slight, mild, moderate and heavy) was determined based on the HI. A clinically significant difference in one analyte was defined as a difference greater than its Clinical Laboratory Improvement Amendments of 1988 (CLIA'88) total allowable error (TAE) limits. The results demonstrated that the mean differences in 11 serum analytes (unconjugated bilirubin, $\mathrm{Ca}^{2+}$, equivalent $\mathrm{CO}_{2}, \mathrm{Cl}^{-}$, creatinine, glucose, $\mathrm{Mg}^{2+}$, phosphorus, $\mathrm{Na}^{+}$, urea nitrogen and uric acid) in hemolyzed and non-hemolyzed samples were within their CLIA'88 TAE limits, while the differences in the other 10 analytes [alanine aminotransferase (ALT), albumin (ALB), amylase (AMYL), aspartate aminotransferase (AST), total bilirubin (TBIL), creatine kinase (CK), CK-myocardial band isoenzyme (CK-MB), lactate dehydrogenase (LDH), $\mathrm{K}^{+}$ and total protein (TP)] in paired samples in at least one of
\end{abstract}

Correspondence to: Professor Dianjun Wei, Department of Clinical Laboratory, Hebei Yanda Hospital, 6 Sipulan Road, Yanjiao Economic and Technological Development District, Langfang, Hebei 065201, P.R. China

E-mail: weidianjun01@163.com

${ }^{*}$ Contributed equally

Key words: hemolysis, hemolytic index, clinical biochemistry test, emergency department, dry slide chemistry analyzer the four groups were greater than their CLIA'88 TAE limits. These results suggest that hemolysis had a notable impact on ALT, ALB, AMYL, AST, TBIL, CK, CK-MB, LDH, $\mathrm{K}^{+}$and TP levels. Furthermore, for ALT, AMYL, TBIL and TP, wet chemistry methods displayed superior anti-hemolytic ability compared with dry chemistry methods. Notably, a high concentration of AST was less affected by hemolysis.

\section{Introduction}

The accuracy of clinical laboratory tests is crucial for decision-making in the diagnostic process. Hemolysis is one of the most frequent pre-analytical errors. The prevalence hemolysis is $3.3 \%$ of all laboratory samples (1) and 6-30\% of blood samples drawn in the emergency department (ED) (2-4). Most hemolysis events occur in vitro in the ED (5), which could be induced by improper techniques during blood collection and processing (6). Hemolysis leads to inaccurate results and often requires repeat testing, which can increase patient discomfort, ED costs and throughput time (5), and thus, hemolysis has a negative impact on the delivery and quality of healthcare. Therefore, it is important to investigate the effects of hemolysis of blood samples in clinical chemistry tests, particularly in samples collected in the ED.

Most automated analyzers can assess the red blood cell (RBC) hemoglobin content, thereby grading the degree of hemolysis, expressed as the hemolytic index (HI), for all plasma/serum samples. The influence of hemolysis on clinical biochemistry tests has been investigated in previous studies, which used three common methods (osmotic shock, freeze or shear) to prepare the hemolysates (6-10). The hemolysates obtained using the latter two methods contain cellular constituents from all types of blood cells (RBCs, leukocytes and platelets), whereas hemolysates obtained using the first method (osmotic shock) only contain cellular constituents from RBCs (11). Although the osmotic shock method was recommended for use in the preparation of hemolysates (11), all three methods mimic the hemolysis that may occur in patient samples. In addition, these studies are almost always performed using wet chemistry analyzers. Given the lack of studies on hemolysis interference using a dry chemistry 
analyzer and the lysis methods, which do not completely reflect true hemolysis in published results (6-10), 92 paired ED blood samples were collected in the present study. Each pair included one hemolyzed sample and one successful (non-hemolyzed) redraw from the same patient, and 21 common laboratory analytes were measured using the $\operatorname{VITROS}^{\circledR} 5600$ dry slide chemistry analyzer. The effects of true hemolysis occurring in patient samples on routine clinical biochemistry tests were evaluated. The results of the present study may help ED clinicians to adjust test results from hemolyzed samples without resampling, thus improving the diagnostic accuracy and enabling timely treatment of patients admitted to the ED.

\section{Materials and methods}

Blood specimen and clinical data collection. A total of 92 pairs of venous blood samples $(3 \mathrm{ml})$ were collected by venipuncture in vacuum tubes from 92 patients admitted to the ED between August, 2018 and December, 2019 at the Second Hospital of Tianjin Medical University (Tianjin, China). One hemolyzed sample was identified by visual detection, another blood sample was immediately drawn from the same patient within $20 \mathrm{~min}$, and the two blood samples constituted one pair. The 92 patients did not receive any drug treatment prior to the second blood collection. Furthermore, the demographic and clinical data of the 92 patients were collected, with a female/male ratio of 48/44, and a median age of 75 years (3-103 years) and preliminary clinical diagnosis (cancer, chronic kidney disease, diabetes mellitus, hypertension, etc.). Written informed consent was obtained from the participating patients, or the parents or guardians of any patients who were under the legal age of consent, and the study protocol was approved by the Research Ethics Committee of The Second Hospital of Tianjin Medical University (approval no. KY2018K092).

Biochemical assays. Each pair of samples was examined within $1 \mathrm{~h}$ after collection. Analyte concentrations and the HI were measured on a VITROS 5600 Dry Slide Chemistry Analyzer (Ortho Clinical Diagnostics, Inc.), which adopts MicroSlide multi-coating film dry sheet technology instead of colorimetric cups for wet chemistry. All serum analytes were measured by dry chemistry, and for analytes that were highly inconsistent with previous studies, the wet chemistry method was used for comparison and verification. The following 21 serum analytes were measured: Alanine aminotransferase (ALT), albumin (ALB), amylase (AMYL), aspartate aminotransferase (AST), total bilirubin (TBIL), unconjugated bilirubin $(\mathrm{Bu}), \mathrm{Ca}^{2+}$, equivalent $\mathrm{CO}_{2}\left(\mathrm{ECO}_{2}\right), \mathrm{Cl}^{-}$, creatine kinase (CK), CK-myocardial band isoenzyme (CK-MB), creatinine (CREA), glucose (GLU), lactate dehydrogenase (LDH), $\mathrm{Mg}^{2+}$, phosphorus (PHOS), $\mathrm{K}^{+}, \mathrm{Na}^{+}$, total protein (TP), urea nitrogen (UREA) and uric acid (URIC). According to descriptive statistics (M, mean; SD, standard deviation; min, minimum; max, maximum) for the HI, four hemolysis levels were determined, including slight hemolysis ( $\mathrm{SH} ; \min \leq \mathrm{HI}<\mathrm{M}-\mathrm{SD})$, mild hemolysis ( $\mathrm{MH} 1 ; \mathrm{M}-\mathrm{SD} \leq \mathrm{HI}<\mathrm{M})$, moderate hemolysis $(\mathrm{MH} 2$; $\mathrm{M} \leq \mathrm{HI}<\mathrm{M}+\mathrm{SD})$ and heavy hemolysis $(\mathrm{HH} ; \mathrm{M}+\mathrm{SD} \leq \mathrm{HI} \leq \mathrm{max})$ groups. Differences in absolute or percentage analyte concentrations in paired serum samples were compared with total allowable error (TAE) for the analytes established by the latest update of Clinical Laboratory Improvement Amendments of 1988 (CLIA'88) (12,13). A clinically significant difference in one analyte was defined as a difference greater than its CLIA'88 TAE limit.

Statistical analysis. The paired samples t-test or Wilcoxon signed-rank test were used for continuous variables, and a $\chi^{2}$ test or Fisher's exact test were used for categorical variables. A two-sided P-value $<0.05$ was considered to indicate statistically significant differences. SPSS version 25.0 (IBM Corp.) was used for all statistical analysis.

\section{Results}

Patients and samples. A total of 92 pairs of blood samples were collected from 92 ED patients. The primary reasons for visiting the ED were fever $(n=39 ; 42.4 \%)$, respiratory disease $(n=26 ; 28.3 \%)$, heart disease $(n=24 ; 26.1 \%)$, and cerebrovascular disease $(\mathrm{n}=11 ; 12 \%)$ (Table I). In addition, three pairs of normal blood samples were collected from 3 of the 92 patients who suffered a syncope, and the concentrations of blood biochemical parameters change rapidly during syncope (GLU, $\mathrm{LDH}$ and $\mathrm{K}^{+}$) were measured and found to have changed significantly (the values depicted as hemolyzed $\rightarrow$ non-hemolyzed): GLU $(n=3 ; 5.39 \rightarrow 6.13,6.63 \rightarrow 5.2,6.58 \rightarrow 4.71), \mathrm{LDH}(\mathrm{n}=1$; $875.3 \rightarrow 1,028.2)$ and $\mathrm{K}^{+}(\mathrm{n}=1 ; 5.6 \rightarrow 6.2)$.

Hemolytic effects on laboratory analytes. The effects of hemolysis on 21 analytes were analyzed according to the four hemolysis levels (SH, MH1, MH2 and HH; Table II). In $\mathrm{SH}, \mathrm{MH} 1, \mathrm{MH} 2$ and $\mathrm{HH}$, the mean differences in 11 serum analytes $\left(\mathrm{Bu}, \mathrm{Ca}^{2+}, \mathrm{ECO}_{2}, \mathrm{Cl}^{-}, \mathrm{CREA}, \mathrm{GLU}, \mathrm{Mg}^{2+}, \mathrm{PHOS}, \mathrm{Na}^{+}\right.$, UREA and URIC) between hemolyzed and non-hemolyzed samples were within their CLIA'88 TAE limits; however, the differences of the other 10 analytes (ALT, ALB, AMYL, AST, TBIL, CK, CK-MB, LDH, $\mathrm{K}^{+}$and TP) in paired samples in at least one of the four groups were greater than their CLIA'88 TAE limits. In SH, MH1, MH2 and HH, the concentrations of $\mathrm{ECO}_{2}$, GLU, CREA and URIC in hemolyzed samples were slightly lower compared with those in non-hemolyzed samples, while the concentrations of $\mathrm{Ca}^{2+}, \mathrm{Mg}^{2+}$ and UREA in hemolyzed samples were slightly higher compared with those in non-hemolyzed samples. In SH, MH1, MH2 and $\mathrm{HH}$ (with the exception of AST and CK-MB in MH1, and of TP in SH), the differences in the levels of AST, CK-MB, $\mathrm{LDH}, \mathrm{K}^{+}$and $\mathrm{TP}$ between paired samples were greater than their TAE limits. The difference of AST in MH1 within its limit was possibly related to a small increase in AST levels between five hemolyzed and non-hemolyzed samples with high concentrations of AST. Of note, AST levels in seven paired samples (non-hemolyzed vs. hemolyzed: $233.07 \pm 63.69$ vs. $219.08 \pm 70.49 ;-6 \%$ ) from 7 of the 92 patients suggested that, in certain samples with initially high concentrations of AST, the increase in AST with hemolysis was not significant. In $\mathrm{MH} 2$ or $\mathrm{HH}$, the differences in the levels of ALT, ALB, AMYL, TBIL and CK between paired samples were greater than their TAE limits, which indicated that only moderate or heavy degrees of hemolysis could have a significant impact on the detection of these analytes. The concentrations of these 
Table I. Clinical characteristics of 92 ED patients, from whom 92 pairs of venous blood samples were collected.

\begin{tabular}{lc}
\hline Characteristic & Value, $\mathrm{n}=92$ \\
\hline Median age (interquartile range), years & $75(57.5-84)$ \\
Sex, n (\%) & \\
Female & $48(52.2)$ \\
Male & $44(47.8)$ \\
Comorbid conditions, n (\%) & \\
Hypertension & $9(9.8)$ \\
Diabetes mellitus & $13(14.1)$ \\
Heart disease & $24(26.1)$ \\
Respiratory disease & $26(28.3)$ \\
Hepatic disease & $11(12)$ \\
Chronic kidney disease & $21(22.8)$ \\
Cerebrovascular disease & $11(12)$ \\
Old cerebral infarction & $3(3.3)$ \\
Cancer & $3(3.3)$ \\
Shock & $4(4.3)$ \\
Acute abdomen & $7(7.6)$ \\
Trauma & $4(4.3)$ \\
Symptom, n (\%) & \\
Fever & $39(42.4)$ \\
Syncope & $3(3.3)$ \\
Emesis & $4(4.3)$ \\
\hline
\end{tabular}

analytes, the differences in which they were greater than their TAE limits, were statistically significantly different between hemolyzed and non-hemolyzed samples (with the exception of $\mathrm{CK}-\mathrm{MB}$ in $\mathrm{SH}$ ). In addition, the concentrations of Bu, PHOS, $\mathrm{AST}, \mathrm{LDH}, \mathrm{K}^{+}$and TP in hemolyzed samples were higher compared with non-hemolyzed samples, and their differences in paired samples gradually increased (with the exception of AST in MH1), with the increase in hemolysis levels partly due to the release of PHOS, AST, LDH and $\mathrm{K}^{+}$from lysed RBCs $(14,15)$. Interestingly, the differences in URIC levels were greater than their TAE limits only in six paired samples, and the corresponding ED patients $(n=6)$ were all women.

\section{Discussion}

Given that the lysis methods in published results (6-10) do not completely reflect true hemolysis, 92 paired ED blood samples were successively collected, each including one hemolyzed sample and one successful (non-hemolyzed) redraw from the same patient within $20 \mathrm{~min}$ in the present study. Taking into consideration that most contemporary biochemical analyzers can provide a $\mathrm{HI}$, the classical hemolysis grading chart was abandoned and replaced directly by the HI value; furthermore, total results analyzed were classified into four groups according to the dispersion degree of HI (16). In addition, as the effect of hemolysis on a particular analyte differs in terms of direction of bias and magnitude of the bias in different instruments (15), it was deemed necessary to build a hemolytic interference threshold in the emergency laboratory using a dry chemistry analyzer, which adopts MicroSlide multi-coating film dry sheet technology instead of colorimetric cups for wet chemistry, in order to compare with wet chemistry analyzers used in previous similar studies $(8-11,17)$. Dry chemistry analyzers have few or no other supporting facilities or devices, and analyses are easily performed in a timely and convenient manner; therefore, their application may be more suitable for emergency clinical biochemistry tests. However, wet chemistry analyzers are more suitable for mass detection of general inpatients and outpatients, due to the considerably lower cost of reagents. In the present study, all 21 serum analytes were measured by dry chemistry, and for analytes (ALT, AMYL, TBIL and TP) that were highly inconsistent with previous studies, the wet chemistry method was used for comparison and verification.

The effects of hemolysis may be categorized into three mechanisms, as described by Lippi et al (1). First, hemolysis may falsely increase blood constituents, such as $\mathrm{K}^{+}, \mathrm{AST}$, PHOS and LDH, as their content in RBCs is high and they are released upon RBC lysis $(14,15)$. Second, hemoglobin released by hemolysis can falsely increase or decrease colorimetric/turbidimetric results for other substances. Hemoglobin absorbs strongly at 415, 540 and $570 \mathrm{~nm}$, and will therefore affect the concentration of analytes, the quantification of which relies on measurements close to these wavelengths, such as TBIL and AMYL, amongst others (1,9). Third, hemolysis can falsely inhibit biochemical reactions, such as the generation of spuriously high $\mathrm{CK}$ activity due to the competitive activity of RBC adenylate kinase (8). Notably, no previous articles had compared the difference between the two methods, most of samples adopted in the present study were collected from old patients. However, the previous studies all used samples from healthy volunteers. Thus, the present study was closer to the actual situation in a hospital.

In the present study, hemolysis had a small impact on the detection of serum parameters of renal function (CREA, UREA and URIC), serum electrolytes and metals $\left(\mathrm{Ca}^{2+}\right.$, $\mathrm{ECO}_{2}, \mathrm{Cl}^{-}, \mathrm{Mg}^{2+}, \mathrm{PHOS}$ and $\mathrm{Na}^{+}$), and $\mathrm{Bu}$ and GLU. The small influence of hemolysis on the readings of these parameters (CREA, UREA, URIC, $\mathrm{Ca}^{2+}, \mathrm{ECO}_{2}, \mathrm{Na}^{+}, \mathrm{Bu}$ and GLU) was also reported by previous studies (8-11). In $\mathrm{HH}$, the difference in PHOS levels between paired samples within its TAE limit was inconsistent with previous reports $(9,10)$, which may be due to the small number of samples in the $\mathrm{HH}$ group in the present study. An increase in CK and CK-MB levels in hemolyzed samples compared with non-hemolyzed samples may reflect a false elevation caused by adenylate kinase release from RBCs $(15,18,19)$. The negative hemolysis interference with ALT in the present study was also inconsistent with previous studies (8-10,20-22), which showed no significant interference of hemolysis with ALT. The AMYL and TP levels increased in samples with hemolysis, and the increases were greater than their TAE limits in the $\mathrm{MH} 2$ and $\mathrm{HH}$ groups. However, previous reports only showed a mild interference of hemolysis with AMYL and TP levels $(8-10,15,19)$. TBIL levels also increased notably in hemolyzed samples compared with non-hemolyzed samples, in accordance with previous studies $(10,11,21,22)$. However, other previous studies only showed a mild interference of hemolysis with TBIL $(8,9,20)$. These inconsistencies between the present and 










previous investigations are likely attributed to the differences between dry and wet chemistry methods. Compared with wet chemistry methods [(AMYL, continuous monitoring method (wavelength, $405 \mathrm{~nm}$ ); TBIL, diazonium salt method (wavelength, $540 \mathrm{~nm}$ ) or vanadate oxidase method (wavelength, $450 \mathrm{~nm}$ )], the wavelengths [AMYL, kinetic method $(540 \mathrm{~nm})$; TBIL, diazonium salt method $(540 \mathrm{~nm})]$ used by dry chemistry methods are all within the region of 510-620 nm, where hemoglobin absorption occurs (23), which may be the primary reason for the interference of hemolysis with these two parameters. Another 20 paired ED blood samples were collected and operated on the same principle to detect ALT and TP simultaneously using dry and wet chemistry methods. Their differences using dry chemistry methods ( $n=20$; ALT: -19.1\%; TP: $14.3 \%$ ) were greater compared with those using wet chemistry methods ( $n=20$; ALT, $-4.44 \%$; TP, $4.8 \%$ ). These two inconsistencies between the present and previous investigations are more likely to originate from the different antihemolytic properties of dry and wet chemistry methods for detecting ALT and TP. Compared with dry chemistry methods, wet chemistry methods may exhibit considerably better anti-interference ability when exposed to hemolyzed samples.

In addition, it was previously indicated that certain comorbidities, such as diabetes mellitus, cancer, multiple sclerosis, the postmenopausal state, and patients undergoing therapeutic interventions and treatments, such as chemotherapy, anticoagulant therapy or injection of contrast media, could increase RBC fragility, which can yield in vitro hemolysis (15). Regrettably, no similar conclusions could be drawn in the present study, which was possibly due to the small sample size.

Additional limitations of the present study included the reliance on the VITROS 5600 Dry Slide Chemistry Analyzer, the fact that most samples were obtained from elderly patients, and the small sample sizes. Further studies are required to increase the number of samples and investigate the mechanisms underlying the hemolysis-mediated changes in blood biochemical parameters.

In summary, the mean differences in the levels of 11 serum analytes $\left(\mathrm{Bu}, \mathrm{Ca}^{2+}, \mathrm{ECO}_{2}, \mathrm{Cl}^{-}, \mathrm{CREA}, \mathrm{GLU}, \mathrm{Mg}^{2+}\right.$, PHOS, $\mathrm{Na}^{+}$, UREA and URIC) between hemolyzed and non-hemolyzed samples were within their CLIA'88 TAE limits, while the differences in another 10 analytes (ALT, ALB, AMYL, AST, TBIL, CK, CK-MB, LDH, K ${ }^{+}$and TP) between paired samples in at least one of the four HI grading groups were greater than their CLIA'88 TAE limits. For ALT, AMYL, TBIL (vanadate oxidase method) and TP, wet chemistry methods appeared to display excellent anti-hemolytic ability compared with dry chemistry methods. Notably, a high concentration of AST was less affected by hemolysis, and the blood levels of certain biochemical parameters (such as GLU, $\mathrm{LDH}$ and $\mathrm{K}^{+}$) may change rapidly during certain symptoms/complications, such as syncope. Hemolysis may impact the results of laboratory measurements, particularly when $\mathrm{HI} \geq \mathrm{MH} 2(\mathrm{HI} \geq 136.97)$; otherwise, more attention must be paid to AST, $\mathrm{LDH}$ and $\mathrm{K}^{+}$levels. Of note, the degree of the influence of hemolysis differs between dry and wet chemistry methods. The results of the present study may assist ED clinicians in improving the pragmatism of disease diagnosis and treatment.

\section{Acknowledgements}

Not applicable.

\section{Funding}

The present study was supported by the Natural Science Foundation of the Second Hospital of Tianjin Medical University (grant no. 2019ydey16) and the Medical Science Research Program of Hebei Province (grant no. 20191066).

\section{Availability of data and materials}

The datasets used and/or analyzed during the present study are available from the corresponding author on reasonable request.

\section{Authors' contributions}

SQL and JL designed the study. SQL and JL wrote the manuscript. SQL, LN and DWW performed the experiments. JL and LN analyzed the results. JL and DJW edited the manuscript. All the authors have read and approved the final manuscript. SQL and LN confirmed the authenticity of all the raw data.

\section{Ethics approval and consent to participate}

The experimental protocol used in the present study was approved by the Ethics Committee for Human Subject Study of The Second Hospital of Tianjin Medical University (Tianjin, China; approval no. KY2018K092). All patients provided written informed consent.

\section{Patient consent for publication}

Not applicable.

\section{Competing interests}

The authors declare that they have no competing interests.

\section{References}

1. Lippi G, Blanckaert N, Bonini P, Green S, Kitchen S, Palicka V, Vassault AJ and Plebani M: Haemolysis: An overview of the leading cause of unsuitable specimens in clinical laboratories. Clin Chem Lab Med 46: 764-772, 2008.

2. Heyer NJ, Derzon JH, Winges L, Shaw C, Mass D, Snyder SR, Epner P, Nichols JH, Gayken JA, Ernst D and Liebow EB: Effectiveness of practices to reduce blood sample hemolysis in EDs: A laboratory medicine best practices systematic review and meta-analysis. Clin Biochem 45: 1012-1032, 2012.

3. Grant MS: The effect of blood drawing techniques and equipment on the hemolysis of ED laboratory blood samples. J Emerg Nurs 29: 116-121, 2003.

4. Söderberg J, Jonsson PA, Wallin O, Grankvist K and Hultdin J: Haemolysis index-an estimate of preanalytical quality in primary health care. Clin Chem Lab Med 47: 940-944, 2009.

5. Phelan MP, Reineks EZ, Schold JD, Hustey FM, Chamberlin J and Procop GW: Preanalytic factors associated with hemolysis in emergency department blood samples. Arch Pathol Lab Med 142: 229-235, 2018 .

6. Zhang WZ and Price DJ: A statistical model for restoration of serum potassium level disturbed by hemolysis. Clin Chim Acta 497: 137-140, 2019. 
7. Perović A and Dolčić M: Influence of hemolysis on clinical chemistry parameters determined with Beckman Coulter tests-detection of clinically significant interference. Scand J Clin Lab Invest 79: 154-159, 2019.

8. Du ZH, Liu JQ, Zhang H, Bao BH, Zhao RQ and Jin Y: Determination of hemolysis index thresholds for biochemical tests on Siemens Advia 2400 chemistry analyzer. J Clin Lab Anal 33: e22856, 2019.

9. Yanagisawa Y, Isobe K, Naito A, Ishijima M, Nanmoku T, Yamamoto T, Suzuki E and Kawakami Y: Influence of in vitro hemolysis on 80 different laboratory tests. Clin Lab 63: 219-226, 2017.

10. Monneret D, Mestari F, Atlan G, Corlouer C, Ramani Z, Jaffre J, Dever S, Fressart V, Alkouri R, Lamari F, et al: Hemolysis indexes for biochemical tests and immunoassays on Roche analyzers: Determination of allowable interference limits according to different calculation methods. Scand J Clin Lab Invest 75: 162-169, 2015.

11. Gidske G, Sølvik UØ, Sandberg S and Kristensen GBB Hemolysis interference studies: Freeze method should be used in the preparation of hemolyzed samples. Clin Chem Lab Med 56: e220-e222, 2018.

12. Goyal T and Schmotzer CL: Validation of hemolysis index thresholds optimizes detection of clinically significant hemolysis. Am J Clin Pathol 143: 579-583, 2015.

13. Centers for Disease Control and Prevention (CDC): Clinical Laboratory Improvement Amendments (CLIA): Clinical laboratory improvement amendments clinical laboratory improvement amendments of 1988 (CLIA) proficiency testing regulations related to analytes and acceptable performance. CDC, Atlanta, GA, 2020. www.cdc.gov/clia/clia-documents. html. Accessed May 28, 2020.
14. Hedayati M, Razavi SA, Boroomand S and Kheradmand KS: The impact of pre-analytical variations on biochemical analytes stability: A systematic review. J Clin Lab Anal 34: e23551, 2020.

15. Simundic AM, Baird G, Cadamuro J, Costelloe SJ and Lippi G: Managing hemolyzed samples in clinical laboratories. Crit Rev Clin Lab Sci 57: 1-21, 2020.

16. Lippi G, Lampus S, Danese E, Montagnana M and Salvagno GL: Values and stability of serum (or plasma) indices in uncentrifuged serum and lithium-heparin plasma. Diagnosis (Berl) 6: 45-47, 2019.

17. Ünlü B, Küme T, Emek M, Örmen M, Doğan Y, Şişman AR, Ergör G and Çoker C: Effect of blood cell subtypes lysis on routine biochemical tests. J Med Biochem 37: 67-77, 2018.

18. Szasz G, Gerhardt W, Gruber W and Bernt E: Creatine kinase in serum: 2. Interference of adenylate kinase with the assay. Clin Chem 22: 1806-1811, 1976.

19. Thomas L: Haemolysis as influence \& interference factor. EJIFCC 13: 95-98, 2002.

20. Lippi G, Salvagno GL, Montagnana M, Brocco G and Guidi GC: Influence of hemolysis on routine clinical chemistry testing. Clin Chem Lab Med 44: 311-316, 2006.

21. Luksic AH, Nikolac GN, Miler M, Dukic L, Bakliza A and Simundic AM: Visual assessment of hemolysis affects patient safety. Clin Chem Lab Med 56: 574-581, 2018.

22. Ji JZ and Meng QH: Evaluation of the interference of hemoglobin, bilirubin, and lipids on Roche Cobas 6000 assays. Clin Chim Acta 412: 1550-1553, 2011.

23. Farage MA, Cambron T and Liu KZ: Visible-near infrared spectroscopic assessment of urogenital tissue in premenopausal and postmenopausal women. Clin Med Insights Womens Health 11: $1179562 \times 17749608,2018$ 